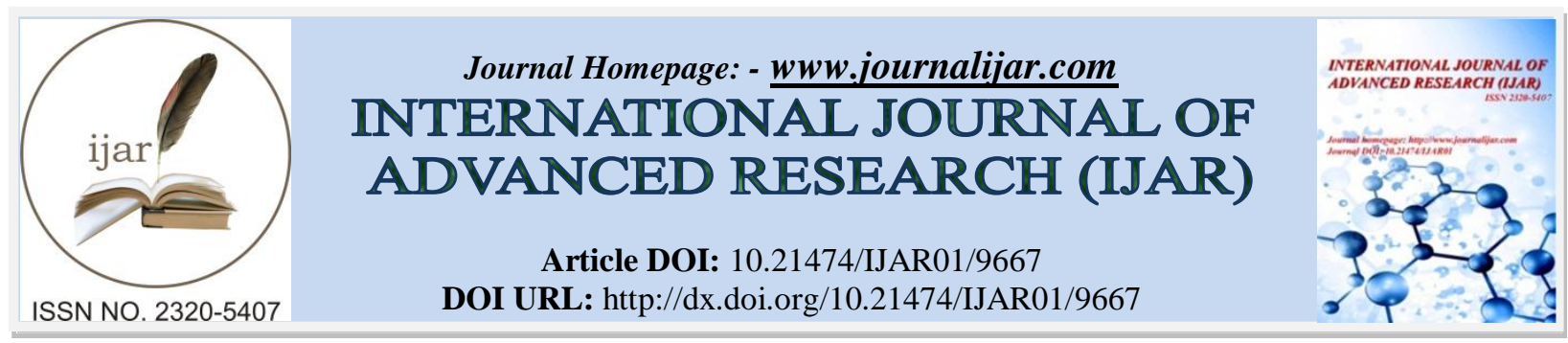

RESEARCH ARTICLE

\title{
INCIDENCE OF WHITE SPOT LESIONS AFTER TREATMENT WITH FIXED ORTHODONTIC APPLIANCES.
}

\author{
Abdul Rahman H. Sabbagh ${ }^{1}$, Mutaz F. Felemban ${ }^{2}$, Ahmed A. Turkistani ${ }^{3}$ and Nayef H. Felemban ${ }^{4}$. \\ 1. BDS , Faculty of Dentistry, Taif University, Taif, Kingdom of Saudi Arabia. \\ 2. BDS , Faculty of Dentistry, Taif University, Taif, Kingdom of Saudi Arabia.. \\ 3. BDS , Faculty of Dentistry, Taif University, Taif, Kingdom of Saudi Arabia. \\ 4. Associate Professor ,Department of Orthodontics, Faculty of Dentistry, Taif University, Taif, Kingdom of Saudi Arabia.
}

\section{Manuscript Info}

Manuscript History

Received: 04 July 2019

Final Accepted: 06 August 2019

Published: September 2019

Key words:-

White spot lesions, Incidence, Dental

Plaque, Demineralization.

\section{Abstract}

Objective: To determine the incidence of white spot lesions (WSLs) in patients undergoing fixed orthodontic treatment for a period of one and two years.

Materials and Methods: The presence of WSLs was assessed in patients at the time of orthodontic application (Group 1) and after one and two years of treatment (Groups 2 and 3, respectively). Presence of WSLs was determined based on clinical intra-oral photographs of the upper four front teeth and scored as present or absent. The frequency of WSLs in each group was calculated and then compared using Fisher's exact test.

Results: In Group 1 (control), WSLs were present in 14 of 48 patients (30\%). In Group 2 (1 year of treatment), WSLs were present in 36 of 48 patients $(75 \%)$. In Group 3 (2 years of treatment), WSLs were present in 38 of 48 patients $(79 \%)$. The frequency of WSLs in Group 1 was significantly different compared to Group $2(P=0.0134)$ and Group $3(P=0.0087)$. However, no significant difference in frequency of WSLs was seen in comparing Groups 2 and $3(P=0.8782)$

Conclusion: More than $70 \%$ of patients who undergo orthodontic treatment develop WSLs, especially in the first months of treatment. This high incidence of WSLs stresses the importance of patient compliance with an oral hygiene protocol that should be tailored for each patient.

Copy Right, IJAR, 2019,. All rights reserved.

\section{Introduction:-}

White spot lesions (WSLs) are areas of demineralized enamel that can develop due to poor oral hygiene. The introduction of orthodontic appliances into the oral cavity increases the risk of developing WSLs. ${ }^{1}$ This increased risk occurs due to the complex design of fixed orthodontic appliances which cause an increased chance of plaque adhesion and further make it more difficult for the patient to clean that area using regular oral hygiene methods. ${ }^{2}$ After the fixation of orthodontic appliances in the oral cavity, a rapid transformation in the bacterial flora of plaque occurs. The amount of acidogenic bacteria in the plaque increases, mostly Streptococcus mutans and species of Lactobacillus. ${ }^{3}$ This increase in acidogenic bacteria results in decreased plaque $\mathrm{pH}$ in orthodontic patients to a greater extent than in non-orthodontic patients. ${ }^{2}$ Therefore, patients undergoing orthodontic treatment have an 
increased risk to develop carious lesions. These lesions are seen most commonly on the buccal surface of the teeth around the brackets, especially adjacent to the gingiva. ${ }^{4}$ These WSLs can develop during the first month of treatment, and if untreated can result in irreversible cavitation. ${ }^{5}$ Zachrisson et al. found that in patients with orthodontic appliances, the labiogingival aspect of the lateral incisors is the most common site for WSLs, and the maxillary posterior segments are the least common site, ${ }^{6}$ and further found that male patients are affected more commonly than female ones. ${ }^{7}$ In a clinical study of orthodontic patients, Tufekci et al. concluded that in the first months of treatment it is critical to evaluate oral hygiene, as WSLs developed rapidly in the first 6 months, and then continued to increase in number at a slower rate over the months to follow. ${ }^{7}$ The published incidence of WSLs among orthodontic patients varies widely from as little as $2 \%$ to as high as $96 \%$, depending on the study. ${ }^{1,4,8}$ This wide variation formed the underlying motivation for this study. The primary goal is to describe the incidence of WSLs after treatment with fixed orthodontic appliances at time of application and after 1 and 2 years of treatment.

\section{Materials And Methods:-}

A cross sectional study was conducted after approval from the dental research ethics committee of the authors' institution. A total of 144 subjects (age mean \pm standard deviation $22 \pm 8$ years) were enrolled in 3 groups of 48 subjects each: Group 1 was the control group and consisted of subjects examined immediately after the application of orthodontic appliances. Group 2 consisted of subjects examined after 1 year of treatment with fixed orthodontic appliances. Group 3 consisted of subjects examined after 2 years of treatment. Inclusion criteria for each group were as follows: all four upper front teeth (FUFT) must be fully erupted and fully visible without structural abnormalities, fillings, veneers or any other type of reconstruction on the buccal side, and at least four brackets must be bonded to the FUFT. Each patient had been given the same oral hygiene instructions and special cleaning instructions including a recommendation for the daily use of fluoride toothpaste and fluoride mouthwash.

Each subject was evaluated for the presence of WSLs using clinical intra-oral photographs. The clinical photographs were taken by various orthodontic consultants. The mode of examination was indirect examination.

The upper four front teeth were examined for WSL and scored as follows: Score 0: No WSLs in any of the four upper front teeth (FUFT). Score 1: Presence of WSLs in any of the four upper front teeth (FUFT).

Statistical analysis was conducted using SPSS v21. The frequency of WSLs in each of the three groups was calculated and then compared using Fisher's exact test, with statistical significance level set at $\mathrm{P}<0.05$.

\section{Results:-}

The number of patients in each group with WSLs is shown in Figure 1. In group 1 (control), 14 of 48 patients (30\%) had WSLs (score 1), and 34 of 48 patients (70\%) had no WSLs (score 0). In group 2 (1 year of treatment), 36 of 48 patients (75\%) had WSLs (score 1), and 12 of 48 patients (25\%) had no WSLs (score 0). In group 3 (2 years of treatment), 38 of 48 patients (79\%) had WSLs (score 1) and 10 of 48 patients (21\%) had no WSLs (score 0 ). When compared using Fisher's exact test, a significant difference was seen in the frequency of WSLs seen in Group 1 relative to both Group $2(\mathrm{P}=0.0134)$ and Group $3(\mathrm{P}=0.0087)$. However, no significant difference was found when comparing the frequency of WSLs in Group 2 and Group 3 ( $\mathrm{P}=0.8782)$.

\section{Figure 1 : Frequency of patients with WSLs.}

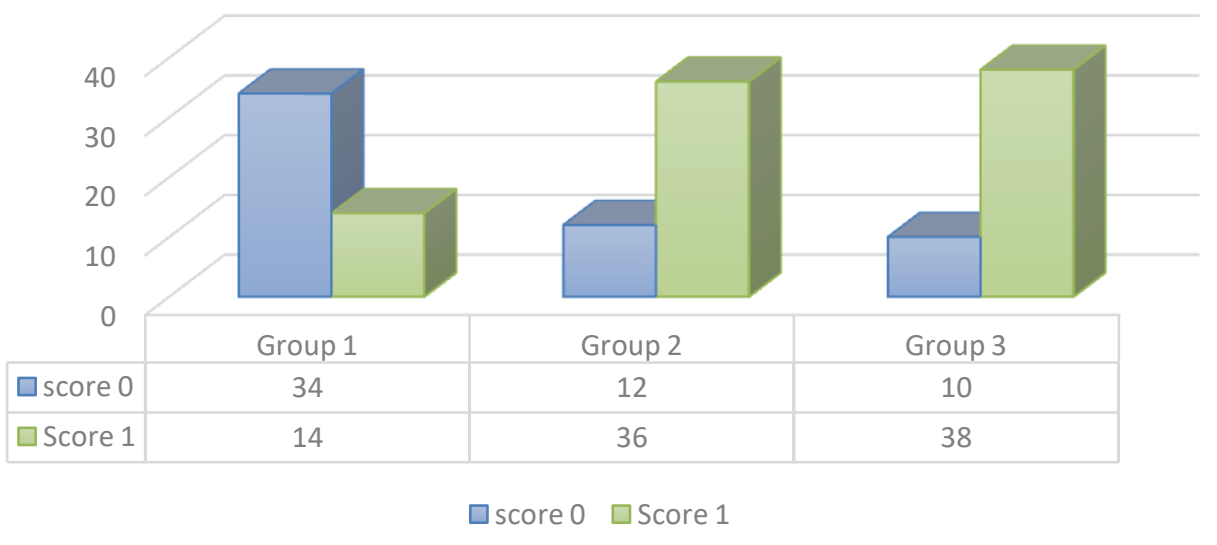




\section{Discussion:-}

Tufekci et al. concluded that the first WSLs started to develop rapidly in the first 6 months then continued to increase in number at a slower rate in the months to follow. ${ }^{6}$ This conclusion correlates well with the findings of the present study, as a significant increase in the prevalence of WSLs was found in the group of patients at 1 and 2 years into orthodontic treatment when compared to the control group observed immediately after orthodontic application. Further, no significant difference in prevalence of WSLs was seen comparing the groups after 1 year and 2 years of treatment. Other studies worldwide have found similar results. Richter et al. found the incidence for WSLs to be approximately $72 \%$ within a sample of 350 subjects undergoing orthodontic treatment over a 12 month period. ${ }^{9}$ Similarly, Sagarika et al. reported an incidence of WSLs of 76\% among 90 subjects over 24 months of orthodontic treatment. ${ }^{10}$ In contrast, other researchers have found lower rates of WSLs, such as Akin et al. and Enaia et al., who reported WSLs incidences of $55 \%$ and $61 \%$, respectively over a treatment period of at least 1 year. ${ }^{11,12}$ These differences may be due to a variety of patient factors that are not considered in this study such as salivary flow, gender, and diet. A lack of accounting for these factors is an important limitation of this study; however, it is to our knowledge the first to measure the incidence rate of WSLs in patients after application of fixed orthodontic appliances in Saudi Arabia.

\section{Conclusion:-}

Most patients (more than 70\%) who undergo fixed orthodontic treatment develop white spot lesions, especially in the first months of the treatment. Despite recent advances, prevention of demineralization during orthodontic treatment is considered one of the greatest challenges for clinicians. Therefore, for each patient an oral hygiene protocol should be tailored with the goal of preventing the development of WSLs. Patient compliance is critical in order to achieve this goal, and so is improved communication among the patient, general dentist, and orthodontist in order to minimize the risk of developing WSLs and to take proper measures once they start to appear.

\section{Conflict of interest}

None.

\section{References:-}

1. Gorelick L, Geiger AM, Gwinnett AJ. Incidence of white spot formation after bonding and banding. Am J Orthod. 1982;8:93-98.

2. Chatterjee R, Kleinberg I. Effect of orthodontic band placement on the chemical composition of human incisor tooth plaque. Arch Oral Biol. 1979;24:97-100.

3. Lundström F, Krasse B. Streptococcus mutans and lactobacilli frequency in orthodontic patients; the effect of chlorhexidine treatments. Eur J Orthod. 1987;9:109-116.

4. Mitchell L. Decalcification during orthodontic treatment with fixed appliances--an overview. Br J Orthod. 1992;19:199-205.

5. Øgaard B. Prevalence of white spot lesions in 19-near-olds: A study on untreated and orthodontically treated persons 5 years after treatment. Am J Orthod Dentofac Orthop. 1989;96:423-427.

6. Tufekci E, Dixon JS, Gunsolley JC, Lindauer SJ. Prevalence of white spot lesions during orthodontic treatment with fixed appliances. Angle Orthod. 2011;81:206-210.

7. Zachrisson BU, Zachrisson S. Caries incidence and oral hygiene during orthodontic treatment. Scand J Dent Res. 1971;79:394-401.

8. Mizrahi E. Enamel demineralization following orthodontic treatment. Am J Orthod. 1982;82:62-67.

9. Richter AE, Arruda AO, Peters MC, Sohn W. Incidence of caries lesions among patients treated with comprehensive orthodontics. Am J Orthod Dentofac Orthop. 2011;139:657-664.

10. Gopikrishna V, Loganathan S, Sagarika N, Suchindran S. Prevalence of white spot lesion in a section of Indian population undergoing fixed orthodontic treatment: An in vivo assessment using the visual International Caries Detection and Assessment System II criteria. J Conserv Dent. 2012;15:104.

11. Akin M, Tazcan M, Ileri Z BF. ncidence of white spot lesion during fixed orthodontic treatment. Turkish $\mathbf{J}$ Orthod. 2013;26:98-102.

12. Enaia M, Bock N, Ruf S. White-spot lesions during multibracket appliance treatment: A challenge for clinical excellence. Am J Orthod Dentofac Orthop. 2011;140:e17-24. 